\title{
Fibromatosis Intraabdominal Mesentérica de Identificación Postmortem. Reporte de caso
}

Post mortem identification of Intra abdominal Mesenteric Fibromatosis.

\section{Case report}

\begin{abstract}
Dr. Edwin Eduardo Velásquez Maldonado ${ }^{1, *}$, Dr. Nicolás Sabillón ${ }^{2}$, Dra. Ana Molina ${ }^{3}$ ${ }^{1}$ Médico Residente del Postgrado de Medicina Legal y Forense, ${ }^{2}$ Especialista en Anatomía Patológica, Dirección de Medicina Forense y Universidad Nacional Autónoma de Honduras, ${ }^{3}$ Médico Autopsiante Dirección de Medicina Forense.
\end{abstract}

\section{REFERENCIA}

Velásquez E, Sabillón N, Molina A. Fibromatosis intraabdominal mesentérica de identificación posmortem. Reporte de caso. Rev. Cienc. Forenses Honduras. 2018; 4(2): 12-16.

Recibido: Abril 2018/ Aceptado:Noviembre 2018.

Los autores declaran que no hay conflicto de intereses

\section{RESUMEN}

La Fibromatosis Intraabdominal es una entidad rara, de etiología desconocida, benigna, sin riesgo de metástasis, pero de comportamiento clínico muy agresivo. Se presenta el caso de femenina de 47 años de edad con paro de evacuaciones de un mes de evolución, marcada distensión abdominal e intolerancia a la vía oral, quien fue manejada en la emergencia de adultos de un hospital público, sin poder determinar su diagnóstico, quien falleció en su casa 30 días después de su última atención hospitalaria. El diagnostico post-mortem en la autopsia médico legal se estableció como Fibromatosis Intraabdominal. Se requiere un alto índice de sospecha ante esta enfermedad con el fin de proveer manejo adecuado a las complicaciones asociadas y disminuir la mortalidad atribuible a ellas.

\section{PALABRAS CLAVE:}

Fibromatosis abdominal, Neoplasia abdominal, Fibromatosis profunda, Tumor desmoide, Reporte de caso.

\section{ABSTRACT}

The intraabdominal fibromatosis is a rare entity, unknown etiology benign, risk of metastasis, but very aggressive clinical behavior. We present the case of a 47-year-old female patient, one-month bowel movements were stopped, marked abdominal distension and intolerance to the oral route, who was managed in the Adult Emergency Department of the public hospital public, unable to determine his diagnosis, who died at home 30 days after his last hospital care. Post-mortem diagnosis refers to intra-abdominal fibromatosis at medical-legal autopsy. It is concluded that a high index of suspicion is necessary before this disease. In order to provide adequate management of the associated complications and decrease the mortality attributable to them"

\section{KEYWORDS}

Abdominal fibromatosis, Abdominal Neoplasms, Deep fibromatosis, Desmoid Tumours; Case Report.

\section{INTRODUCCIÓN}

El término de fibromatosis, propuesto por Stout, se designa para un grupo de condiciones relacionadas que tienen características en común. La fibromatosis intraabdominal (FIA) a nivel mundial se presenta aproximadamente en 1 por 1,000,000/año. En Honduras se ha publicado solamente un estudio de 28 casos de fibromatosis y solo uno correspondió a FIA; 
reportando que las mujeres presentan mayor predisposición al padecimiento en relación a los hombres ${ }^{1}$.

La fibromatosis intraabdominal mesentérica (FIAM) está comprendida dentro del grupo de las fibromatosis, es el tumor primario más común del mesenterio y representa aproximadamente el $8 \%$ de todas las fibromatosis ${ }^{1,2}$. De carácter benigno, con crecimiento localmente agresivo e infiltración de los planos adyacentes ${ }^{3}$, con comportamiento clínico agresivo, escasa incidencia y asociada a una alta tasa de morbimortalidad. Macroscópicamente se observa proliferación de fibroblastos, patrón de crecimiento infiltrativo, presencia de colágeno entre células proliferantes, falta de características citológicas de malignidad, actividad mitótica escasa/ausente y carencia de metástasis ${ }^{3-7}$. El cuadro clínico depende de la extensión e invasión del tumor, puede cursar asintomática durante un período de tiempo y caracterizarse por tener una presentación clínica muy variada, cuando la invasión es extensa y la enfermedad se encuentra activa pueden desarrollarse complicaciones como obstrucción intestinal, ureteral, perforación de víscera hueca o hidronefrosis, entre otras. El diagnóstico de la FIAM se basa en un alto índice de sospecha clínica, coadyuvado por estudio de imágenes y confirmado por histopatología ${ }^{8,9}$. Se presenta el caso de paciente que falleció a causa de obstrucción intestinal secundaria a FIAM.

Este reporte resalta la importancia de proveer un manejo holístico a los pacientes con sintomatología concordante, a fin de prevenir fallecimientos y sus posibles repercusiones legales.

\section{LÍNEA DE TIEMPO}

$\begin{array}{cl}\text { Atencion } & \begin{array}{l}\text { Ingresó al } \\ \text { Médica } 1\end{array} \\ \text { Hospital } \\ 19 / 6 / 2013 & 3 / 7 / 2013\end{array}$

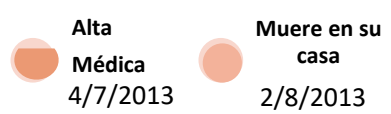

\section{PRESENTACIÓN DE CASO}

Paciente femenina, 47 años de edad, con historia de sufrir dolor abdominal leve, náuseas, vómitos e hiporexia; motivo por el que es llevada a emergencia de un hospital, donde se evaluó y se decidió manejo ambulatorio. Al empeorar su condición fue llevada nuevamente a la emergencia del hospital, debido al cese de evacuaciones, distensión abdominal, fiebre, náuseas, vómitos e hiporexia; ingresó nuevamente al hospital, refiriendo los familiares desconocer el diagnóstico, tratamiento y manejo realizado, se otorgó el alta médica al día siguiente, a pesar de su escasa mejoría. El cuadro clínico de la paciente se deterioró cada vez más, se incrementó la intensidad del dolor y la distensión abdominal e intolerancia a la vía oral, la paciente no buscó nuevamente atención médica, con detrimento clínico, falleció en su casa de habitación, 28 días después de su último ingreso hospitalario. Debido a que no se pudo establecer el diagnóstico antemortem y a las circunstancias en que falleció, se le realizó autopsia médico legal, para establecer la causa y manera de muerte.

\section{HALLAZGOS RELEVANTES DE AUTOPSIA}

1- Cicatriz antigua en región abdominal.

2.-Marcada distensión abdominal por ascitis de aproximadamente $8,000 \mathrm{ml}$ de líquido color amarillo claro.

3- Masa tumoral localizada en mesenterio, de color blanco - amarillo, de consistencia dura comprometiendo la pared de intestino delgado y colón, con obstrucción parcial de la luz. (Figura $1 \mathrm{~A}$ y $1 \mathrm{~B})$.

4.-Microscópicamente el tumor estaba constituido por proliferación de fibroblastos, sin atipia, con fibrosis difusa, afectando la serosa y la muscular propia del intestino delgado y del colón. (Figura 1C) 
Figura 1: Vista macroscópica y microscópica de la FIAM

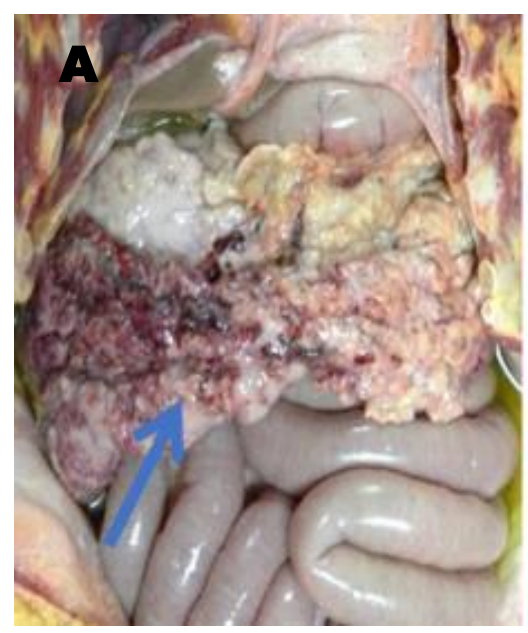

Figura 1: fibromatosis mesentérica intrabdominal

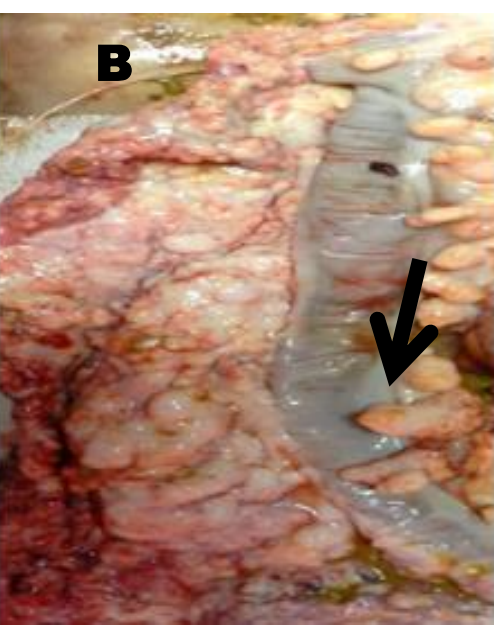

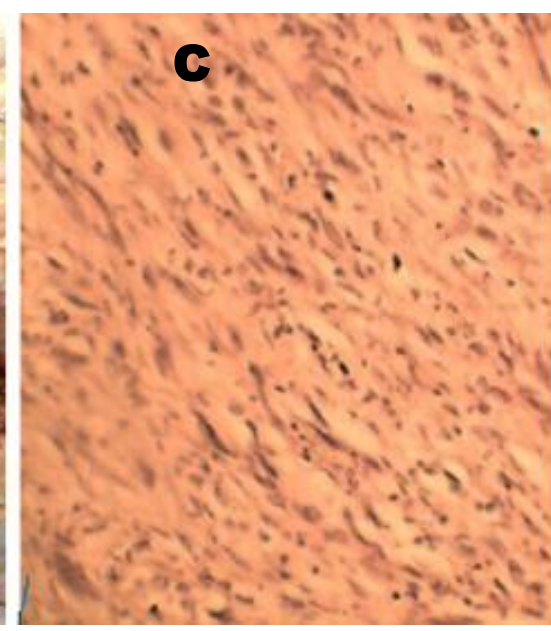

(A) Tumor en mesenterio (Fecha). (B) Tumor comprometiendo intestino, como lo indica la flecha (C) Corte histológico del tumor, tinción HE 10X

\section{DISCUSIÓN}

Este el primer caso de FIAM diagnosticado en autopsia médico legal, abordado por la Dirección de Medicina Forense de Honduras; el caso derivó hasta autopsia debido a que la muerte ocurrió en la casa de habitación, sin un diagnóstico médico previo y en circunstancias aparentemente sospechosas de mal manejo, según la percepción de los familiares.

La etiología de la fibromatosis intraabdominal es desconocida, sin embargo, los estudios publicados indican que hay factores de riesgo como: cirugías o traumatismos abdominales previos, embarazo, sexo femenino, tratamiento con estrógenos, antecedentes familiares, poliposis adenomatosa familiar y ciertas alteraciones genéticas, como trisomía del cromosoma 8 y $20^{9}$.

Se presenta con más frecuencia entre los 40 y 60 años, en adultos del sexo femenino, que han tenido uno o más hijos ${ }^{10}$. Otros autores sin embargo encontraron una relación hombre/mujer de 3 a 1 , en individuos que se encontraban entre la tercera o cuarta década de la vida. En nuestro caso la paciente, estaba en la quinta década de la vida, y presentaba una cicatriz quirúrgica antigua, en la línea media del abdomen, no pudiéndose establecer la causa del proceso quirúrgico, todos estos hallazgos son compatibles con lo descrito por otros autores. Aunque clínicamente la FIAM, es una enfermedad que se presenta en la mayoría de los casos en forma asintomática, con un discreto dolor abdominal, sin masa palpable, observándose con menor frecuencia sangrado o abdomen agudo secundario a perforación de víscera hueca ${ }^{10}$. Cuando forman tumores, estos suelen manifestarse como una masa cuyos síntomas dependen de la localización. En la región abdominal, lo habitual es que ocasionen dolor y distensión abdominal, obstrucción intestinal o ureteral, sangrado o perforación de víscera hueca, o permanecen asintomáticos durante mucho tiempo, favoreciendo el diagnóstico tardío ${ }^{11-12}$, como lo observado en este caso donde la paciente presentó un comportamiento clínico silencioso inicial y posteriormente agresivo y de rápida evolución, que se manifestó como un cuadro obstructivo intestinal, se le dio manejo inespecífico sin 
ninguna mejoría de los síntomas, inexplicablemente el expediente no consigna que se le haya realizado estudio de imágenes. El diagnóstico de la FIAM se establece mediante estudios de imagen, como tomografías y ultrasonido, con manejo quirúrgico, para la resección completa de la sección afectada o quimioterapia, esto siempre y cuando se realice el diagnóstico oportuno. De forma definitiva el diagnostico se establece mediante el análisis histopatológico de muestras de tejido obtenidas al momento de la resección quirúrgica o postmortem ${ }^{7}$. Microscópicamente, las lesiones están pobremente circunscritas, con infiltración del tejido blando circundante. La microscopia de alto poder demuestra la apariencia uniforme de células elongadas en forma de huso. El colágeno separa estas células, resultando un mínimo contacto intercelular. ${ }^{14,15}$

En el caso presentado, posiblemente debido a la falta de sospecha clínica, no se realizaron estudios de imagen, el diagnóstico definitivo de la enfermedad fue hecho post-mortem a través de hallazgos macroscópicos y microscópicos de alteraciones en epiplón, mesenterio e intestino. Histológicamente se observó proliferación de fibroblastos, sin atipia, con patrón de crecimiento infiltrativo, tal como lo reporta la literatura ${ }^{16}$.

La autopsia médico-legal permitió establecer la FIAM, como causa de muerte y esclarecer el desarrollo clínico de la enfermedad, la cual fue de inicio asintomático, pero a posteriori curso con un cuadro evidentemente clínico, hasta llevar a un desenlace fatal, como lo fue el choque hipovolémico secundario a obstrucción intestinal, se desconoce porque la paciente no busco atención médica nuevamente.

\section{REFERENCIAS BIBLIOGRÁFICAS}

1. Goldblum J, Weiss S, Folpe AL. Mesenteric fibromatosis. [Internet]. En: Goldblum J,
Weiss S, Folpe AL. Enzinger and Weiss's Soft

Tissue Tumors [Internet]. 6th ed.

Philadelphia: Saunders; 2013. [citado 12

noviembre 2018]. Disponible en:

https://www.elsevier.com/books/enzinger-

and-weisss-soft-tissue-

tumors/goldblum/978-0-323-08834-3

Disponible en:

https://www.ncbi.nlm.nih.gov/nlmcatalog/1

\section{$\underline{01464289}$}

2. Alvarado D, Ferrera C. Fibromatosis reporte de 28 casos y actualización del tema. Rev Med Hondur [Internet]. 1988 [Consultado 12 noviembre 2018];56(2):158-166. Disponible en:

http://www.bvs.hn/RMH/pdf/1988/pdf/Vol5 6-2-1988-7.pdf

3. Whittle C, Sánchez L, Albarracin J, Hepp J. Fibromatosis Mesentérica: caso clínicoimagenológico y revisión bibliográfica. Rev Méd Chile [Internet]. 2006 [Consultado 12 noviembre 2018];134(1):85-9. Disponible en: https://scielo.conicyt.cl/pdf/rmc/v134n1/art 12.pdf

4. Barotto $M$, Rivoira G, Parodi $M$, Napolitano D, Viscido G, Doniquian Marcelo, et al . Fibromatosis mesentérica: Reporte de un caso. Rev Chil Cir [Internet]. 2008 [Consultado 12 noviembre de 2018];60(6):557-60. Disponible en: https://scielo.conicyt.cl/pdf/rchcir/v60n6/art 14.pdf

5. Rosai A. Fibromatosis. In : Ackerman LV, Rosai J. Rosai and Ackerman's surgical pathology. $10^{\text {th }}$ ed. Philadelphia PA: Elsevier; 2011. 2 vol. p.2116.

6. Retamozo S, Brito-Zerón $\mathrm{P}$, Bosch $\mathrm{X}$, Almirall AS, Villalta J, et al. Fibrosis Retroperitoneal y mesenteritis "idiopáticas" (Enfermedad de Ormond): ¿Posible enfermedad relacionada con 
IGG4? Evaluación histórica de 22 casos.

Rev Clin Española [Internet]. 2014 [Consultado 12 de noviembre de 2018];214 (Espec Congr):

$724 . \quad$ Disponible en:

http://www.revclinesp.es/controladores Lcongresos-

7. Meneses Calderón J, Moreno Santillán AA, González Díaz JI, Díaz de León Ponce MA, Ortega Chimal R, García Cueva G, et al. Fibromatosis mesentérica retroperitoneal: reporte de un caso y revisión de la literatura. Rev Asoc Mex Med Crit y Ter Int [Internet]. 2008 [Consultado 12 noviembre 2018];22(1):4245. Disponible en: www.medigraphic.com/pdfs/medcri/ti2008/ti081h.pdf

8. Palacios-Zertuche JT, Cardona-Huerta $S$, Juárez-García ML, Valdés-Flores $\mathrm{E}$, Muñoz-Maldonado GE. Reporte de caso: tumor desmoides gigante de pared abdominal con rápido crecimiento durante el embarazo. Cir [Internet]. 2017 [Consultado 12 noviembre 2018];85(4):339-343. Disponible en: http://www.sciencedirect.com/science/ article/pii/S0009741116300366

9. $D M$, Ghalige $H S, R S$, Sharma $M B$, Singh TS. Mesenteric fibromatosis (desmoid tumour) - a rare case report. J Clin Diagn Res [Internet]. 2014 [Consultado 12 noviembre 2018];(11):ND01-2.

Disponible en:

https://www.ncbi.nlm.nih.gov/pmc/artic les/PMC4290279/

10. Burke AP, Sobin LH, Shekitka KM, Federspiel $\mathrm{BH}$, Helwig EB. Intra-abdominal fibromatosis. A pathologic analysis of 130 tumors with comparison of clinical subgroups. Am J Surg Pathol [Internet]. 1990 [Consultado 12 noviembre 2018];14(4):335-41. Disponible en: https://www.ncbi.nlm.nih.gov/pubmed/ 2321698

11. Molloy AP, Hutchinson B, O'Toole GC. Extraabdominal desmoid tumours: a review of the literature. Sarcoma [Internet]. 2012 [Consultado 12 noviembre 2018];578052. Disponible en:

https://www.hindawi.com/journals/sarc oma/2012/578052/

12. Papagelopoulos PJ, Mavrogenis AF, Mitsiokapa EA, Papaparaskeva KT, Galanis EC, Soucacos PN. Current trends in the management of extra-abdominal desmoid tumours. World J Surg Oncol [Internet]. 2006 [Consultado 12 noviembre 2018];4(21). Disponible en: https://www.ncbi.nlm.nih.gov/pubmed/ $\underline{16584569}$

13. Garzón A, Díaz MC, Fernández-Ávila DG, Gutiérrez JM. Fibrosis retroperitoneal idiopática. Acta Med Colomb [Internet]. 2015 [Consultado 12 noviembre 2018];40(3):260$62 . \quad$ Disponible en: http://www.scielo.org.co/pdf/amc/v40n 3/v40n3a15.pdf

14. González M, Menéndez R, Ayala JM, Herrero $M$, Cuesta J, Domínguez A, et al. Tumor desmoide intrabdominal. Cir Esp [Internet]. 2005 [Consultado 12 de noviembre 2018];77(6): 362-4. Disponible en: http://www.elsevier.es/es-revistacirugia-espanola-36-pdf-13075726

15. Dolz-Aspas R, Moragrega-Cardona B, VilarBonacasa E, Gracia-Sánchez P. Fibrosis retroperitoneal, enfermedad de Ormond. Casos clínicos con diferente presentación y revisión de la literatura. Nefrología Sup Ext [Internet]. 2012 [Consultado 12 noviembre 2018];3(4):84-7. Disponible en: http://www.revistanefrologia.com/es-pdf$\underline{\mathrm{X} 2013757512000857}$ 